\title{
NEW INTERPRETATION OF THE ATOMIC SPECTRA OF THE HYDROGEN ATOM: A MIXED MECHANISM OF CLASSICAL LC CIRCUITS AND QUANTUM WAVE-PARTICLE DUALITY
}

\section{NUEVA INTERPRETACIÓN DEL ESPECTRO ATÓMICO DEL ÁTOMO DE HIDRÓGENO: UN MECANISMO MIXTO DE CIRCUITOS LC Y LA DUALIDAD ONDA CUÁNTICA-PARTÍCULA}

\author{
H. Torres-Silva ${ }^{1}$ \\ Recibido el 5 de septiembre de 2007, aceptado el 12 de diciembre de 2007 \\ Received: September 5, $2007 \quad$ Accepted: December 12, 2007
}

\begin{abstract}
RESUMEN
En este trabajo se presenta un estudio de las leyes macroscópicas de conversión de energía del oscilador armónico LC, la onda electromagnética (fotones) y el átomo de hidrógeno. Como nuestro análisis indica, las energías de estos aparentemente diferentes sistemas obedecen exactamente la misma ley de conversión de la energía. Sobre la base de nuestros resultados y de la dualidad onda-partícula del electrón, nos encontramos con que el átomo de hidrógeno, de hecho, es un oscilador LC microscópico naturalmente quiral.

En el marco de la teoría clásica de campos electromagnéticos se obtiene analíticamente, para el átomo de hidrógeno, el radio cuantizado de la órbita electrónica $r_{n}=a_{o} n^{2}$ y la energía cuantizada $E_{n}=-R_{H} h c / n^{2},(\mathrm{n}=1,2,3 .$.$) , donde a_{0}$ es el radio de Bohr y $R_{H}$ es la constante de Rydberg. Sin la adaptación de otros principios fundamentales de la mecánica cuántica, se presenta una explicación razonable de la polarización de los fotones, las reglas de selección y principio de exclusión de Pauli. Los resultados también ponen de manifiesto una conexión esencial entre el espín de electrón y el movimiento helicoidal intrínseco de los electrones e indican que el espín es el efecto de un confinamiento cuántico.
\end{abstract}

Palabras clave: Átomo de Bohr, quiralidad, oscilador LC.

\begin{abstract}
In this paper we study the energy conversion laws of the macroscopic harmonic LC oscillator, the electromagnetic wave (photon) and the hydrogen atom. As our analysis indicates, the energies of these apparently different systems obey exactly the same energy conversion law. Based on our results and the wave-particle duality of electrons, we find that the hydrogen atom is, in fact, a natural chiral microscopic LC oscillator.

In the framework of classical electromagnetic field theory we analytically obtain, for the hydrogen atom, the quantized electron orbit radius ${ }_{n}=a_{o} n^{2}$, and quantized energy $E_{n}=-R_{H} h c / n^{2},(n=1,2,3, \cdots)$, where $a_{0}$ is the Bohr radius and $R_{H}$ is the Rydberg constant. Without the adaptation of any other fundamental principles of quantum mechanics, we present a reasonable explanation of the polarization of photon, selection rules and Pauli exclusion principle. Our results also reveal an essential connection between electron spin and the intrinsic helical movement of electrons and indicate that the spin itself is the effect of quantum confinement.
\end{abstract}

Keywords: Bohr atom, chirality, LC oscillator.

\section{INTRODUCTION}

No one doubt that twentieth century is the century of quantum theory [1-10]. After 100 years of development quantum physics is no longer just a field, it is the bedrock of all of modern physics. Although the modern quantum theory has provided a beautiful and consistent theory for describing the myriad baffling microphenomena which had previously defied explanation [3], one should not neglect a curious fact that quantum mechanics

1 Instituto de Alta Investigación. Universidad de Tarapacá. Antofagasta No 1520. Arica, Chile. E-mail: htorres@uta.cl 
never take into account the deep structures of atoms. In fact, at the heart of quantum mechanics lies only the Schrödinger equation [5], which is the fundamental equation governing the electron. According to quantum theory, it is the electromagnetic interaction (by the exchange of photons) which hold electrons and nuclei together in the atoms.

But, up to now, quantum theory never provides a practical model of how electron and nuclei can absorb and emit photons.

In this paper, we investigate the energy relationship of electron in the hydrogen atom. Significantly, we find a process of perfect transformation of two forms of energy (kinetic and field energy) inside the atom and the conservation of energy in the system. By applying the principle of wave-particle duality and comparing to known results of the macroscopic harmonic LC oscillator and microscopic photon, we are assured that electron kinetic energy in fact is a kind of magnetic energy and the atom is a natural microscopic LC oscillator. Moreover, the mixed mechanism (classical LC circuits / quantum wave particle duality) turns out to have remarkably rich and physical properties which can used to describe some important quantum principles and phenomena, for instance, polarization of photon, Zeeman effect, Selection rules, the electron's mass and spin, zero point energy (ZPE), the Pauli exclusion principle.

\section{ENERGY TRANSFORMATION AND CONVERSION IN HYDROGEN ATOM}

Classically, as shown in figure 1, the hydrogen atom consists of one electron in orbit around one proton with the electron being held in place via the electric Coulomb force. Equation of motion is

$$
\frac{e^{2}}{4 \pi \varepsilon_{0} r^{2}}=\frac{m_{e} u^{2}}{r}
$$

where $m_{e}$ is mass of electron. Eq. 1 can be rewritten in the form of kinetic energy $E_{k}$ and field energy $E_{f}$ (stored in the capacitor of hydrogen atom) as follows:

$$
\frac{e^{2}}{2 C_{r}}=\frac{1}{2} m_{e} u^{2}
$$

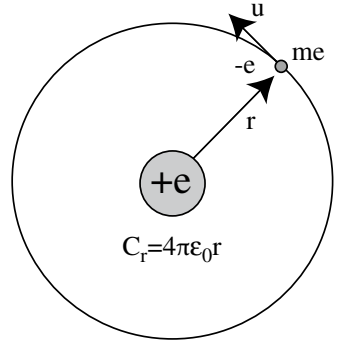

Figure 1. The diagram illustrating the hydrogen atom.

where $C_{r}=4 \pi \varepsilon_{0} r$ is the capacitance of the hydrogen system. Thus the total energy of the hydrogen system is given by

$$
T_{\text {total }}=\left|\frac{1}{2} m_{e} u^{2}-\frac{e^{2}}{4 \pi \varepsilon_{0} r}\right|=\frac{e^{2}}{2 C_{r}}
$$

It should be pointed out that Eq. 2 and 3 are the foundation of our study. These two equations together indicate a process of perfect periodically transformation of two forms of energy (kinetic energy $E_{k}=\frac{1}{2} m_{e} u^{2}$ and field energy $E_{f}=\frac{e^{2}}{2 C_{r}}$ inside the atom and the conservation of energy in the system

$$
E_{\text {total }}=E_{f}=E_{k}
$$

Recall the macroscopic harmonic LC oscillator where two forms of energy, the maximum field energy $E_{f}=\frac{Q_{0}^{2}}{2 C}$ of the capacitor $\mathrm{C}$ (carrying a charge $Q_{\mathrm{o}}$ ) and the maximum magnetic energy $E_{m}=\frac{I_{0}^{2}}{2 L}$ of the inductor L, are mutually exactly interconvertible ( $E_{\text {total }}=E_{f}=E_{k}$ ) with a exchange periodic $T=2 \pi \sqrt{L C}$. And for a microscopic photon (electromagnetic wave), the maximum field energy $E_{f}=\frac{1}{2} \varepsilon_{0} E_{0}^{2}$ and the maximum magnetic energy $E_{m}=\frac{1}{2} \mu_{0} \boldsymbol{H}_{0}^{2}$ also satisfy $E_{\text {total }}=E_{f}=E_{k}$ (See appendix A about $E_{f}=-E_{m}$ ).

Based on the above energy relationship for three totally different systems and the requirement of the electromagnetic interaction (by exchanging photon) between electron and nuclei, we assure that the kinetic energy of electron (Eq. 2) is a kind of magnetic energy and the hydrogen atom is a natural microscopic LC oscillator. 
In 2000, a multinational team of physicists had observed for the first time a process of internal conversion between bound atomic states when the binding energy of the converted electron becomes larger than the nuclear transition energy $[11,12]$. This observation indicate that energy can pass resonantly between the nuclear and electronic parts of the atom by a resonant process similar to that which operates between an inductor and a capacitor in an LC circuit. These experimental results can be considered a conclusive evidence of reliability of our LC mechanism.

Here raise an important question: how can the electron function as an excellent microscopic inductor?
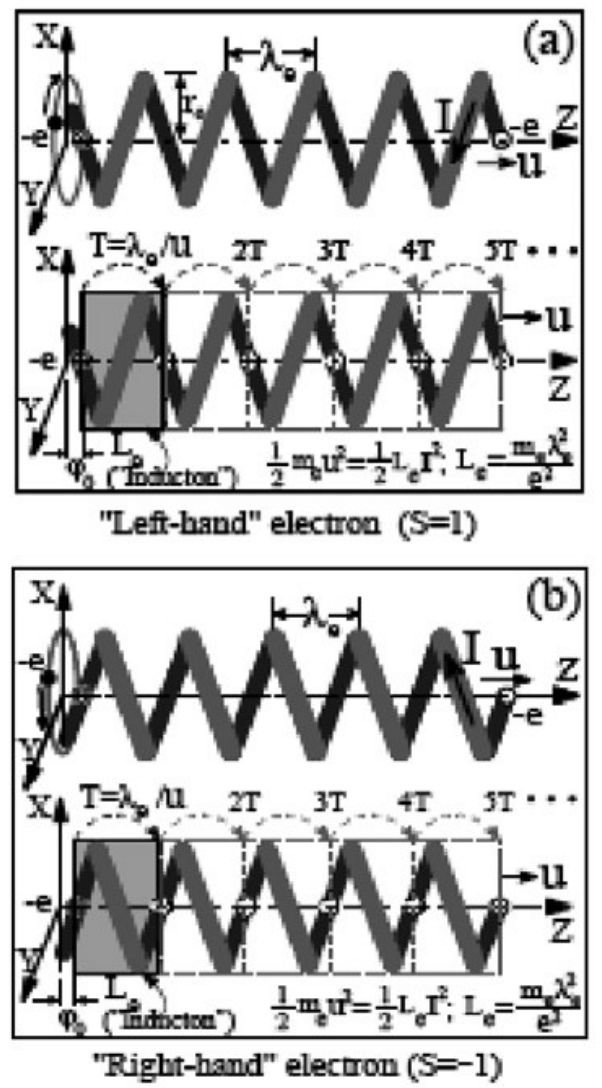

Figure 2. A free electron moving along a helical orbit with a helical pitch of de Broglie wavelength $\lambda_{e}$.

The answer lies in the intrinsic wave-particle duality nature of electron. In our opinion, the wave-particle nature [7] of electron is only a macroscopic behavior of the intrinsic helical motion of electron within its world.

\section{CHIRALITY AND "INDUCTON" OF FREE ELECTRON}

In 1923, Broglie suggested that all particles, not just photons, have both wave and particle properties [5]. The momentum wavelength relationship for any material particles was given by

$$
\lambda=h / p
$$

where $\lambda$ is called de Broglie wavelength, $h$ is Planck's constant [1] and $\mathrm{p}$ the momentum of the particle. The subsequent experiments established the wave nature of the electron $[9,10]$. Eq. 5 implies that, for a particle moving at high speed, the momentum is large and the wavelength is small. In other words, the faster a particle moves, the shorter is its wavelength. Furthermore, it should be noted that any confinement of the studied particle will shorten the $\lambda$ and help to enhance the so-called quantum confinement effects.

As shown in figure 2 (a) and (b), based on Eq. 5 and the demanding that the electron would be a microscopic inductor, we propose that a free electron can move along a helical orbit (the helical pitch is de Broglie wavelength $\left.\lambda_{e}\right)$ of left-handed or right-handed. In this paper, the corresponding electrons are called "Left-hand" and "Right-hand" electron which are denoted by Chirality Indexes $S=1$ and $S=-1$, respectively. Hence, the electron can now be considered as a periodic-motion quantized inductive particle which is called "inducton" (see figure 2). Moreover, the particle-like kinetic energy of electron can be replaced with a dual magnetic energy carried by a "inducton". Therefore, we have

$$
E_{k}=\frac{1}{2} m_{e} u^{2}=\frac{1}{2} L_{e} I^{2}
$$

where $\mathrm{u}$ is the axial velocity of the helical moving electron and $L_{e}$ is the inductance of the quantized "inducton".

The above relation indicates that the mass of electron is associated with an amount of magnetic energy. From figure 2, the electric current, for one de Broglie wavelength, is given by

$$
I=\frac{e u}{\lambda_{e}}
$$

From Eq. 7, it is important to note that the electric current should be defined within an integral number of de Broglie 
wavelength. Hence, the electric current $I=\frac{e u}{2 \pi r}$ (where $r$ is the electronic orbital radius in the hydrogen atom), which was widely used in the semiclassical Bohr model, may be physically invalid. Collecting Eq. 6 and 7 together, we have the inductance of single "inducton"

$$
L_{e}=\frac{m_{e} \lambda_{e}^{2}}{e^{2}}
$$

Then the dual nature of electron can be uniquely determined by $L_{e}$, the periodic T (or frequency $f=\frac{1}{T}=\frac{u}{\lambda_{e}}$ ), the initial phase $\varphi_{0}$ and the chirality $(S=1$ or $S=-1)$.

\section{ATOMIC SPECTRA OF HYDROGEN ATOM}

Quantized radius and energy by the application of helical electron orbit to the hydrogen atom (figure 2), we can explain the stability of the atom but also give a theoretical interpretation of the atomic spectra. Figure 3 shows four possible kinds of stable helical electron orbits in hydrogen atom, and each subgraph corresponds to a electron of different motion manner within the atom. The electrons can be distinguished by the following two aspects. First consider the chirality of electron orbits, as shown in figure 3, the electrons of figure 3(a) and (c) are "Left-hand" labelled by $S=1$, while electrons of figure 3(b) and (d) are "Right-hand" labelled by $S=-1$. Secondly consider the direction of electron orbital magnetic moment $\mu \mathrm{L}$, figure 3(a) and (b) show that the $\mu \mathrm{L}$ are in the $\mathrm{Z}$ direction (Up) while (c) and (d) in the $-Z$ direction (Down), the corresponding electrons are labelled by $\mathrm{J}=1$ and $\mathrm{J}=-1$, respectively, here $J$ is called Magnetic Index. Hence, the electrons of different physical properties become distinguishable, they are Up "Left-hand" (ULH) electron $(\mathrm{J}=1, \mathrm{~S}=1)$, Up "Right-hand" (URH) electron ( $=1, S=-1)$, Down "Left-hand" (DLH) electron ( $\mathrm{J}=-1, \mathrm{~S}=1)$ and Down "Right-hand" (DRH) electron $(\mathrm{J}=-1, \mathrm{~S}=-1)$.

As shown in figure 3(a), the helical moving electron around the orbit mean radius $r$ can now be regarded as a quantized "inducton" of $\lambda_{r}$, thus the hydrogen atom is a natural microscopic LC oscillator.

We consider that the physical properties of the hydrogen atom can be uniquely determined by these natural LC parameters. To prove that our theory is valid in explaining the structure of atomic spectra, we study the quantized
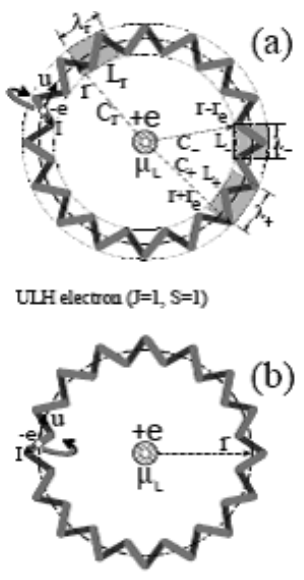

URH electron $(\mathrm{J}=1, \mathrm{~S}=-1)$

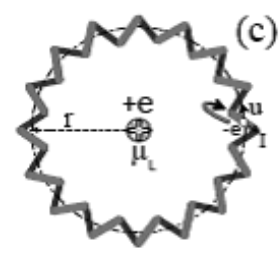

DLH electroa $(\mathrm{J}=-1, \mathrm{~S}=1)$

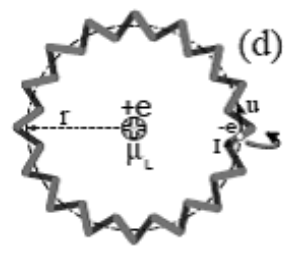

DRH electron ( $(\mathrm{f}=-1, \mathrm{~S}=-1)$
Figure 3. The quadruple degenerate stable helical electron or-bits in hydrogen atom.

orbit radius and the quantized energy of hydrogen atom and make a comparison between our results of LC mechanism and the known results of quantum theory. For the system of $\lambda_{r}$, the LC parameters of the hydrogen atom is illustrated in figure 3 . Then the LC resonant frequency is

$$
v_{r}=\frac{1}{2 \pi \sqrt{L_{r} C_{r}}}
$$

Recall the well-known relationship $E=h v_{r}$, we have

$$
E=h v_{r}=\frac{e^{2}}{8 \pi \varepsilon_{0} r}
$$

Combining Eq. 9 and Eq. 10 gives

$$
\lambda_{r}=\frac{2 h}{e} \sqrt{\pi \varepsilon_{0} r / m_{e}}
$$

Then the stable electron orbits are determined by

$$
\frac{2 \pi r}{\lambda_{r}}=n,(\mathrm{n}=1,2,3 \cdots),
$$

where $\mathrm{n}$ is called Principal oscillator number. The integer $\mathrm{n}$ shows that the orbital allow integer number of "induction" of the de Broglie wavelength $\lambda_{r}$. From Eq. 11 and Eq. 12, the quantized electron orbit mean radius is given by 


$$
r_{n}=\frac{\varepsilon_{0} h^{2}}{\pi m_{e} e^{2}} n^{2}=a_{0} n^{2}
$$

where $\alpha_{0}$ is the Bohr radius. And the quantized energy is

$$
E_{n}=-\frac{e^{2}}{8 \pi \varepsilon_{0} r_{n}}=-\frac{m_{e} e^{4}}{8 \varepsilon_{0}^{2} h^{2}} \frac{1}{n^{2}}=-R_{H} \frac{h c}{n^{2}}
$$

where $R_{H}$ is the Rydberg constant. Surprisingly, the results of Eq. 13 and 14 are in excellent agreement with Bohr model [3]. Besides, taking figure 3 into account, we can conclude that the quantized energies of Eq. 14 are quadruple degenerate.

\section{CONCLUDING REMARKS}

In conclusion, we have found a process of perfect transformation of two forms of energy (kinetic and field energy) inside the hydrogen atom and the conservation of energy in the system. Then, we have shown that the helical moving electron can be regarded as a inductive particle ("inducton") while atom is regarded as a microscopic LC oscillator, then the indeterministic quantum phenomena can be well explained by the deterministic classical theory. For a microscopic photon (electromagnetic wave), the maximum field energy $E_{f}=\frac{1}{2} \varepsilon_{0} E_{0}^{2}$ and the maximum magnetic energy $E_{m}=\frac{1}{2} \mu_{0} \boldsymbol{H}_{0}^{2}$ are connected so $\boldsymbol{E}=i \boldsymbol{H}$ (see equation (A11) of appendix A). The vector Poyting vanishes and the Hydrogen atom does no radiate and it is stable. In particular, with this approach we can show another phenomena such how a pairing Pauli electron can move closely and steadily in a DNA-like double helical electron orbit. Moreover, we can have pointed out that the mass of electron, the ZPE and what has been called the intrinsic "electron spin" are all really the quantum confinement effects of the intrinsic chirality of the electron of helical motion.

We have shown that the quantum mechanism is nothing but an electromagnetic theory (with the radius of the helical orbit $r_{e} \rightarrow 0$ ) of the LC/wave-particle duality mixed mechanism. Our mixed mechanics force us to rethink the nature and the nature of physical world. We believe all elementary particles, similar to photon and electron, are only some different types of energy representation.
Though, the standard quantum mechanics nature is intrinsically probabilistic, permitting only predictions about probabilities of the occurrence of an event.

Nevertheless, one century after its birth, it still presents many unclarified issues at its very foundations. Starting from an Einstein's work [13], many attempts have been devoted to build a deterministic theory reproducing all the results of quantum mechanics. The latter include the de Broglie-Bohm's hidden variable theory, the most successful attempt in this sense [14]. Recently, a first experimental test of de Broglie-Bohm theory against standard quantum mechanics was reported [15]. In our study, it has been shown definitely that the electron follows a perfectly defined trajectory in its motion, which confirms the de Broglie-Bohm's prediction. Also in our work, it is found that the known wave-particle duality can be best manifested by showing that the wave motion associated with a electron is just the phenomenon of its complex helical motion in real space. Therefore, the wave-particle duality should lie at the heart of the quantum universe.

We are now more and more convinced that the universe was built in the simplest manner and all things in it are unique and definitive. As Albert Einstein one said, "God does not play dice with the universe". Of course, a more clear understanding of microscopic world is still of the greatest challenge.

\section{APPENDIX A}

It is generally believed that in transverse electromagnetic waves electric field $\mathbf{E}$ and magnetic field $\mathbf{B}$ are always perpendicular to each other. In this Letter we show that, however, a general class of transverse electromagnetic waves with $\mathbf{E} \| B$ exists in a chiral media. We show how to obtain these waves in general and give example in vacuum and plasma . All these waves carry magnetic helicity. In a cold collision less chiro-plasma, the magnetostatic mode $[16,17]$ of this class becomes the more familiar force-free field $\nabla \times \mathbf{B}=k \mathbf{B}, k=\sqrt{k_{0}^{2}+\mu T}$ where $T$ is the chiral parameter. We consider transverse electromagnetic waves in a uniform medium. These transverse waves can be described by

$$
\begin{gathered}
\mathbf{B}=\nabla \times \mathbf{F}, \\
\mathbf{E}=-\frac{1}{c} \frac{\partial \mathbf{F}}{\partial t},
\end{gathered}
$$


in which the vector potential $\mathbf{F}$ satisfies $\nabla \cdot \mathbf{F}=0$ and the wave equation

$$
\nabla \times \nabla \times \mathbf{F}+\frac{1}{c^{2}} \frac{\partial^{2} \mathbf{F}}{\partial t^{2}}=\frac{4 \pi}{c}(\mathbf{j}+\beta \nabla \times \mathbf{J})
$$

Here

$$
\mathbf{j}=\vec{\sigma} \cdot \mathbf{E}
$$

where $\vec{\sigma}$ is the conductivity tensor operator of the medium under consideration, After Fourier analysis in time, we have

$$
\nabla \times \nabla \times \mathbf{F}-\left(\omega^{2} / c^{2}\right) \vec{K}(\omega) \cdot \mathbf{F}=0
$$

with the dielectric tensor

$$
\ddot{K}(\omega)=\vec{I}-4 \pi \vec{\sigma}(\omega) / i \omega
$$

For simplicity, we consider only cases where $\vec{K}(\omega)$ is independent of wavelength. We first look at the Hydrogen atom in vacuum $\sigma=0, T=\omega / c$ and $\mathbf{j}+\beta \nabla \times \mathbf{J}=0$ and Eq (A3) becomes

$$
\left(\nabla^{2}+k_{0}^{2}\right) \mathbf{F}_{k}=0
$$

with $\omega^{2}=k_{0}^{2} c^{2}$. This waves equation allows the well known linear polarized plane waves with $\mathbf{E}\|\mathbf{B}\| \mathbf{F}$ [18]. For every solution of Eq. (A5), it is straightforward to show that

$$
\mathbf{F}_{k}=\mathbf{A}_{k}+\mathrm{k}_{0}^{-1} \nabla x \mathbf{A}_{k}
$$

satisfies not only Eq. (A5) but also

$$
\nabla \times \mathbf{F}_{k}=k \mathbf{F}_{k}
$$

For those vector potentials $\mathbf{F}, T k_{0}=1$ satisfying Eq. (A7), the electric field $\mathbf{E}$ and magnetic field $\mathbf{B}$ are parallel to each other and both are perpendicular to the vector $\boldsymbol{k}_{0}$. Therefore, for every plane wave solution, a wave solution with $\mathbf{E} \| \mathbf{B}$ can be constructed with $\mathbf{k}=k_{0}(0,0,1)$, so

$$
\begin{gathered}
\mathbf{F}=A\left(\sin k_{0} z, \cos k_{0} z, 0\right) \cos \omega t, \\
\mathbf{E}=(\omega A / c)\left(\sin k_{0} z, \cos k_{0} z, 0\right) \sin \omega t,
\end{gathered}
$$

and

$$
\mathbf{B}=k_{0} A\left(\sin k_{0} z, \cos k_{0} z, 0\right) \cos \omega t
$$

This solution corresponds to two circularly polarized waves [16] propagating opposite to each other in such a way that their Poynting vectors are cancelled out, so

$$
\mathbf{E}=\mathrm{icB}=\mathrm{i} \eta \mathbf{H}
$$

Therefore, a single helical photon with energy $\hbar \omega$ carries a magnetic helicity of hc, and

$$
E_{f}=E_{\boldsymbol{E}}=\frac{1}{2} \varepsilon_{0} \boldsymbol{E}_{0}^{2}=-\frac{1}{2} \mu_{0} \boldsymbol{H}_{o}^{2}=E_{\boldsymbol{H}}=E_{m}
$$

\section{ACKNOWLEDGEMENT}

The author would like to thank to Instituto de Alta Investigación (IAI) for the support of this work.

\section{REFERENCES}

[1] M. Planck. Ann. Phys. Vol. 1, p. 69. 1900.

[2] A. Einstein. Ann. Phys. Vol. 17, p. 132. 1905.

[3] N. Bohr, Phil. Mag. Vol. 26, p. 576. 1913.

[4] O. Stern, Z. Phys. Vol. 2, p. 49. 1920.

[5] E. Schrodinger. Ann. Phys. Vol. 79, p. 361. 1923.

[6] L. de Broglie, Phil. Mag. Vol. 47, p. 446. 1924.

[7] W. Pauli. Z. Phys. Vol. 31, p. 373. 1924.

[8] W. Heisenberg Z. Phys. Vol. 43, p. 172. 1927. 
[9] G.P. Thomson, Proc. Roy. Soc. Vol. 117, p. 600. 1928.

[10] C.N. Yang, Selection Rules for the Dematerialization of a Particle into Two Photons, Phys. Rev. Vol. 77, pp. 242-245. 1950.

[11] T. Carreyre. Phys. Rev. C 62. 2000.

[12] S. Kishimoto. Phys. Rev. Lett. Vol. 85, p. 1831. 2000.

[13] F. Reines and W. H. Sobel. "Test of the Pauli Exclusion Principle for Atomic Electrons". Phys. Rev. Lett. Vol. 32, p. 954. 1974.
[14] D. Bohm. Phys. Rev. Vol. 85, p. 166. 1952.

[15] G. Brida. J. Phys. B 35, p. 4751. 2002.

[16] C. Chu and T. Ohkawa. Phys Rev. Lett. 48, p. 837. 1982.

[17] H. Torres-Silva. "Chiro-plasma surface waves". A. Priou et al editors: Advances in Complex Electromagnetics Materials, Kruwer Academic Pub. Vol. 28, pp. 249-258. 1997.

[18] H. Torres-Silva. Pramana Journal of Physics. Vol. 48, p. 67. 1997. 